\title{
ONLINE DATABASE FOR THE ASSESSMENT AND COMPARATIVE EVALUATION OF REHABILITATION OUTCOMES
}

\author{
T.E. Ward ${ }^{1}$, B. Caulfield ${ }^{2}$ \\ ${ }^{1}$ Department of Electronic Engineering, National University of Ireland, Maynooth, Ireland \\ ${ }^{2}$ School of Physiotherapy, University College Dublin, Dublin, Ireland
}

\begin{abstract}
The changing face of rehabilitation structures and practices has meant that measurement of outcomes of rehabilitation has become an absolute necessity. Unfortunately the level of agreement over which measures to use is poor as a result of lack of access to measures and lack of knowledge concerning methods of analysis and interpretation of results. In this paper we report on efforts by the authors to address these issues through the establishment of an online database available over a variety of web devices for the comparative evaluation of rehabilitation outcomes. We discuss the background to the problem, the development and use of an initial system and the future directions of the project.

Keywords - Outcome measures, Internet, database
\end{abstract}

\section{INTRODUCTION}

Rehabilitation outcome measures are used by clinicians to measure the efficacy and progress of treatment on patients. They often consist of a series of questions the responses to which can be analyzed such that a measure of progress can be ascertained. This work has been developed as a result of previous experiences in carrying out rehabilitation outcome measurement research. [1]. These experiences revealed that levels of standardized outcome measures usage was very low indeed across the EU. In addition, there was very little agreement amongst clinicians as to which outcome measures should be used in clinical practice. These findings were very disappointing, as the changing face of rehabilitation structures and practices has meant that measurement of outcomes of rehabilitation has become an absolute necessity [2]. Subsequent experiences [3] have revealed that clinicians recognise the great need for outcome measurement to become a routine part of their clinical practice yet feel that there are significant barriers to this becoming a reality. Reasons offered for this include:

- Pressures of time.

- Lack of access to measures.

- Lack of knowledge concerning which measures to use in specific circumstances.

- How these measures should be used.

- How to score measures and interpret results.

There is a great need for novel interventions to address these issues in rehabilitation. Clinicians need to be provided with a tool, which enables them to introduce outcome measurement programs to their practice without causing major disruption to their clinical routine.
This paper reports on progress being made by the authors to address these issues through the establishment of an online database for the assessment and comparative evaluation of rehabilitation outcomes.

\section{METHODOLOGY}

\section{A. Clinical Methodology}

The methodology of the final system can be outlined as follows:

1. To select appropriate outcome measurements for use in the areas of orthopaedics/rheumatology, neurology and cardiology. This will be achieved through means of userworkshops, literature review and the results of an ongoing survey of UK and lrish rehabilitation departments.

2. The design and construction of suitable Internet-based interfaces for database data-enty, retrieval and visualisation. 3. Development of disease-specific data collection protocols. 4. Pilot data-collection and user testing phase.

5. Refinement and Optimization

6. Data collection phase comprising of 12 months of entry of outcome measure data by users.

7. Development of data visualisation tools based on userspecifiable criteria.

\section{B. Technological Methodology}

Fig. 1 illustrates the three-tier web-database architecture used in our system. It comprises the database server, the web server, and the browser or client computers. The purpose of the database server is to run the database management system (DBMS) to process Structured Query Language (SQL) statements and perform database management tasks. The web server performs two important tasks. It acts as an hypertext transfer protocol (HTTP) server, receiving requests and generating responses in HTTP format. The server also allows server side scripting so that code can be written in languages like VBScript and JavaScript. It is through these scripting languages that the creation, reading, updating, and deletion of database entries is possible. In database applications, the browser serves three functions. First, the browser is an HTTP client, generating requests for data. It also hosts a scripting environment for executing scripts on the client machine (client side scripting, in this case JScript). Finally, the browser renders HTML, VML or other markup languages into a display in the client browser window.

The HTTP server in this case is IIS (Internet Information Services 5.0) IIS provides an interface called Internet Server Application Program Interface (ISAPI) by which other programs can trap and process HTTP messages. The Active Server Processor (ASP) is one such program. It processes all 
web pages with the suffix asp. When IIS receives such a page, it sends it to Active Server Processor over the ISAPI interface. ASP then processes the page and sends a response back to the client via the ISAPI interface to IIS [4].

ASP hosts scripting languages; therefore, ASP pages can contain JavaScript, VBScript, Perl, ColdFusion and other scripting language statements. These statements will be executed when ASP processes the page. In addition, ActiveX controls can be embedded in the page and they will be invoked as well. VBScript is the language used for the ASP implementation in this system

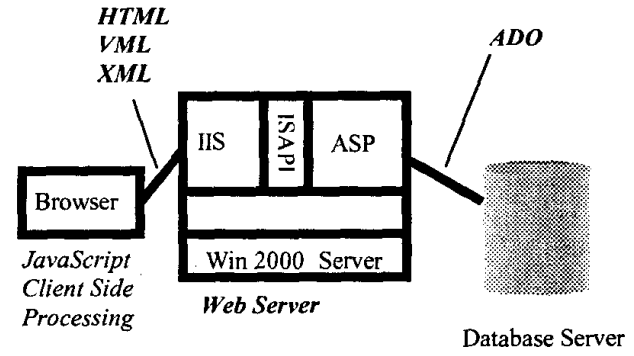

Fig. 1. Three-tier architecture for web application

The database access standard used here is ADO (Active Data Objects). This is a simple object model that can be used by data consumers to interact with a database. It can be called from scripting languages like JScript and VBScript, from Visual Basic, Java and $\mathrm{C}++$. It provides a simpler and cleaner interface than traditional technologies such as ODBC or by making native calls.

\section{RESULTS}

\section{A. Current System}

We have established an interactive website which allows users to compile patient population profiles online using established standardized outcome measurement instruments. Currently these include:

- Barthel Index

- Roland disability Questionnaire (RDQ)

- Customer Satisfaction Questionnaire (CSQ)

Users simply tick the appropriate boxes on the online forms, which are then processed with a simple click of a button to yield a patient score. The user has the options of printing this page for their own records and submitting their score to the database for collation and research purposes.

This system can afford the clinician the opportunity to easily engage in an outcome measurement program and have their patient population results and statistics compiled in an errorfree environment. The current system is in use by a small number of clinicians in a pilot study format. Fig. 2 shows some user screens. Presentation of results can occur through the use of charts generated on demand using Vector Markup Language (VML). VML is an application of Extensible
Markup Language (XML) 1.0 which defines a format for the encoding of vector information together with additional markup to describe how that information may be displayed and edited. VML used in conjunction with ASP allows data to be retrieved from the database and graphical charts to be presented to the clinician. These charts represent a summary of patient progress and can be immediately printed/stored for clinical records. Fig 3. Shows a VML plot summarizing some data.

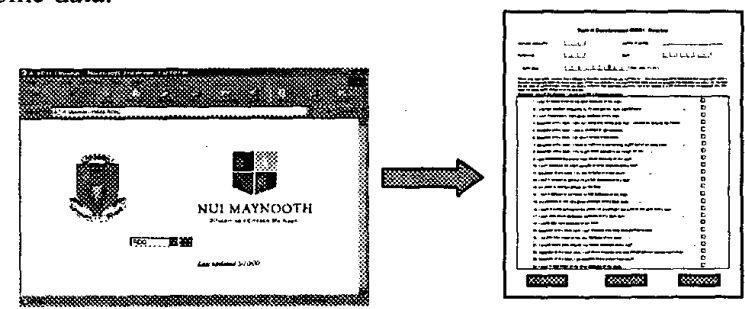

Fig.2, Outcome Measure Selection and data entry

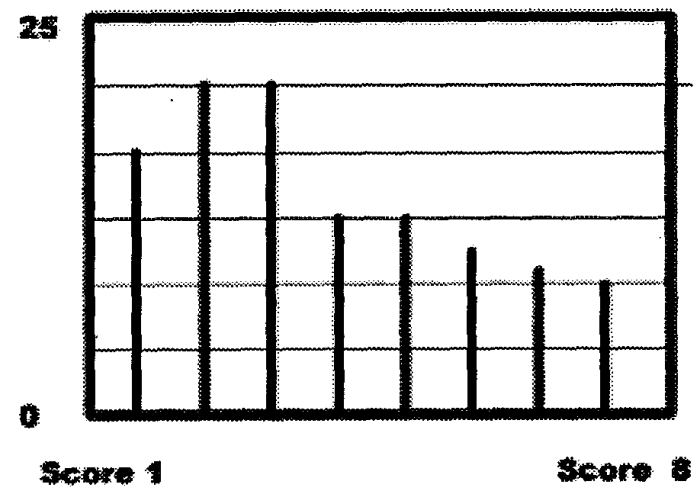

Fig. 3. Typical VML rendering of outcome records.

\section{DISCUSSION}

The current system is fulfilling the role of a trial system in that we are obtaining feedback that will shape future directions of the system. Ultimately we wish to enhance the system further so that it can accept clients over nontraditional networked devices such as mobile clients through WAP or indeed Voice XML. VoiceXML is an application of XML which, when combined with voice recognition technology, enables interactive access to the Web through an ordinary telephone. An individual session works through a combination of voice recognition and keypad entry. This will allow clinicians to conduct these tests in an outpatient scenario without having to use PCs.

The possibilities of such a system as being described here are immense. It is hoped to add additional scales to the current system to provide a wider range of services. It is also possible to integrate additional patient data for more advance 
analysis. It should also be appreciated that by using such a centralized system a great deal of patient data can be assembled. This presents a wonderful opportunity for future researchers in the area of outcome measurement to analyze and assess new and existing outcome measures.

\section{CONCLUSION}

In this paper we have given an account of the design and implementation of an online database for the assessment and comparative evaluation of rehabilitation outcomes. This in so far as the authors are aware is the first such time such an approach has been taken to such a problem and certainly represents an advance in the state-of-the-art in rehabilitation outcome measurement.

\section{REFERENCES}

[1] B. Caulfield, M Garrett, M. Torenbeek and W van Harten W (Eds), Rehabilitation Outcome Measures in Europe: The state of the art. Roessingh $\mathrm{R} \& \mathrm{D}$, Enschede, the Netherlands, 1999.

[2] R. Hammond, "Why an outcome measures database?" Physiotherapy, vol. 85, pp. 234-235, 1999

[3] M. Torenbeek, B. Caulfield, W. van Harten, M:. Garrett, "ACROSS: Current use of Outcome Measures in Europe," International Journal of Rehabilitation Research 2001; vol 24 (in press)

[4] T. Yager, Windows 2000 Web Applications Developer's Guide, Microsoft Technologies Series, NJ, Prentice Hall, 2000. 